\title{
ESTIMACIONES DE LA POBLACIÓN ACTUAL (ePOBa) A NIVEL MUNICIPAL. DISCREPANCIAS CENSO-PADRÓN A PEQUEÑA ESCALA
}

\author{
Francisco J. Goerlich Gisbert \\ Universidad de Valencia e Instituto Valenciano de Investigaciones Económicas
}

\section{RESUMEN}

El INE publica dos tipos de cifras de población: (i) Estimaciones, con desagregación geográfica hasta nivel provincial y basadas en los niveles del último Censo, y (ii) cifras oficiales basadas en el Padrón, un registro administrativo disponible a nivel municipal.

El propósito de este trabajo es doble: (i) Poner de manifiesto las discrepancias a nivel municipal entre las cifras del Censo de 2001 y las del Padrón, y (ii) examinar la posibilidad de construir estimaciones de población a nivel municipal consistentes, en los niveles, con las Estimaciones de Población Actual que publica el INE para las provincias.

Palabras clave: población, municipios, Censo, Padrón, Movimiento Natural de la Población, defunciones, nacimiento, migraciones.

\section{ABSTRACT}

The INE publishes two types of population figures: (i) Population estimates at a provincial level, based on the last Census, and (ii) official population figures based on the administrative record of the Municipal Register of Inhabitants, available at municipal level.

Our purpose is twofold: $(i)$ to show the discrepancies between the population figures of Census 2001 and the registry in 2002, and (ii) exploring the possibilities of building population estimates for municipalities consistent in their levels with the Now Cast population estimates published by INE at regional level.

Key words: population, municipalities Census, Administrative registry, deaths, births migrations.

Fecha de recepción: julio 2010.

Fecha de aceptación: febrero 2012. 


\section{INTRODUCCIÓN}

Las fuentes de información para el estudio de los fenómenos demográficos es un tema recurrente de investigación en el ámbito de las ciencias sociales (Melón 1951, Arango 1981, Reher y Valero 1995, Puyol 1997, Reher 1997, Goerlich, Mas, Azagra y Chorén 2006). Este trabajo pretende ser una contribución en este sentido desde el punto de vista de las fuentes de información actuales sobre la población española.

En la actualidad el Instituto Nacional de Estadística (INE) publica dos tipos de cifras de población: ${ }^{1}$

(i) Por una parte, estimaciones de población (cifras inter-censales o corrientes) y proyecciones (a corto y largo plazo). Estas se publican con un nivel de desagregación geográfico provincial (NUTS 3), están ancladas en los niveles proporcionados por los censos (que tienen lugar cada 10 años) y toman en consideración la información disponible sobre flujos demográficos (nacimientos, muertes y migraciones), ya sea medidos o estimados. Desde el punto de vista del presente trabajo lo que realmente importa aquí es que las estimaciones de población actual ( $e P O B a$ en la terminología del INE) ${ }^{2}$ toman como base para los niveles de población el último censo disponible (actualmente el de 2001).

(ii) Por otra parte, cifras de oficiales de población (en el sentido legal del término) basadas en un registro administrativo cuya formación, mantenimiento, revisión y custodia corresponde al Ayuntamiento de cada municipio, el Padrón Municipal, ${ }^{3}$ si bien el INE ejerce una necesaria tarea de supervisión y coordinación para asegurar la consistencia del sistema. Con el actual sistema de gestión padronal (el llamado Padrón continuo) se obtiene una Revisión del Padrón Municipal con referencia al 1 de enero de cada año, siendo la primera Revisión del Padrón, de acuerdo al actual sistema de gestión, la referenciada a 1 de enero de 1998. Son estas cifras las que acaban convirtiéndose en la población oficial de cada municipio del territorio nacional mediante Real Decreto, si bien de la explotación estadística del Padrón se derivan cifras de población a nivel infra-municipal, ya sea a nivel de sección censal o para todas las unidades poblacionales que aparecen en el nomenclátor. ${ }^{4}$ Desde el punto de vista del presente trabajo lo que realmente importa aquí es que las cifras de población derivadas

1 Véase la nota divulgativa del INE: ¿Qué tipos de cifras de población publica el INE?, en la web del INE: http://www.ine.es/daco/daco43/epoba/cifras.pdf (consultado el 19/4/2010).

2 La metodología para la elaboración de las estimaciones de población actual está descrita en INE (sin fecha, 2010), http://www.ine.es/metodologia/t20/t2030259.htm, documentos que recogen tanto la metodología inicial como la actualmente vigente (consultado el 19/4/2010). Un mayor detalle metodológico puede encontrarse en INE (2009a).

Básicamente las ePOBa se construyen mediante el método de componentes, y a nivel regional incluyen ajustes territoriales a partir de una escala provincial, lo que permite captar comportamientos territoriales diferenciados. Así pues, desde el punto de vista regional adoptan una aproximación top-down, es decir se construyen hacia abajo dadas unas estimaciones nacionales.

3 Las cuestiones metodológicas básicas y la normativa aplicable en relación al Padrón están descritas en http://www.ine.es/metodologia/t20/t203024566.htm (consultado el 19/4/2010).

4 Las cuestiones metodológicas esenciales en relación a las unidades poblacionales que aparecen en el nomenclátor están descritas en http://www.ine.es/nomen2/Metodologia.do (consultado el 19/4/2010). 
Gráfico 1

POBLACIÓN TOTAL: PADRÓN VERSUS CENSO/ESTIMACIONES-INTERCENSALES/ePOBa

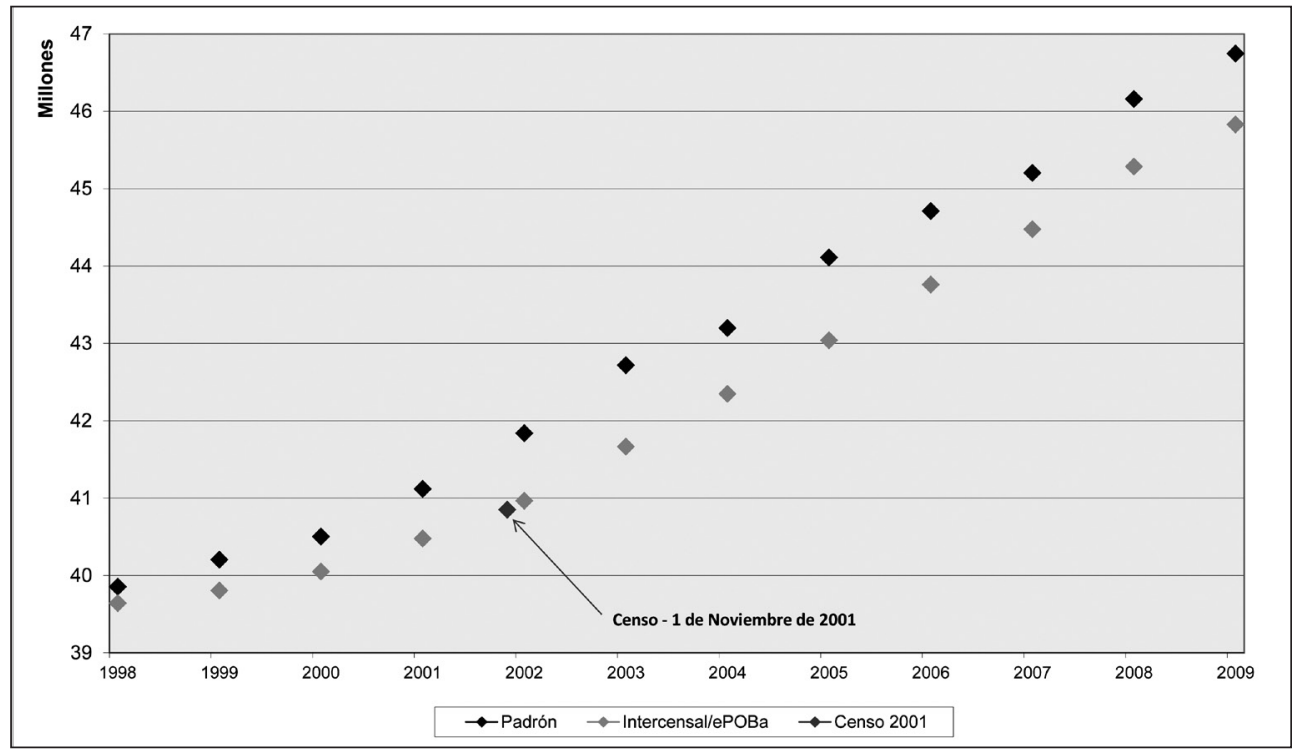

Fuente: Elaboración propia a partir del Censo de 2001, las estimaciones intercensales, la ePOBa y el Padrón municipal.

del Padrón proceden de un registro administrativo sujeto a condicionantes legales y cuya corrección sólo puede efectuarse a nivel de dato individual concreto. ${ }^{5}$

Es bien conocido que ambos tipos de cifras de población difieren de forma importante, las estimaciones de población actual $(e P O B a)$ y las cifras de población derivadas del Padrón no coinciden en sus niveles (Garcia Coll y Sanchez Aguilera 2001; Goerlich 2007). Esta discrepancia se debe en última instancia a que no se hizo coincidir ambas cifras sobre la base del último Censo disponible, como era costumbre con anterioridad a la introducción del Padrón continuo, cuando una de las dos Renovaciones Padronales que debían llevar a cabo los Ayuntamientos cada década, la correspondiente al año terminado en uno, se hacía coincidir con la elaboración del correspondiente Censo (así sucedió con los censos de 1981 y 1991), y ambas cifras se hacían coincidir. De hecho, la última Renovación Padronal de acuerdo al antiguo sistema, que siempre implicaba cierto grado de «recuento» de la población cada 5 años, tuvo lugar el 1 de mayo de 1996.

El gráfico 1 muestra claramente la discrepancia entre los dos tipos de cifras de población. Es obvio que la divergencia crece en los últimos años del siglo $\mathrm{XX}$, tras el último «recuento», y se muestra relativamente estable en la primera década del siglo XXI. En estos últimos años el crecimiento en ambas cifras de población es similar, pero la discrepancia de nivel permanece estable.

5 La naturaleza del Padrón hace que las cifras de población derivadas a partir del mismo adopten, necesariamente, una aproximación bottom-up, es decir las cifras agregadas se obtengan mediante agregación de los datos individuales. 
Gráfico 2

DIFERENCIA EN POBLACIÓN TOTAL: PADRÓN-INTERCENSALES/ePOBa

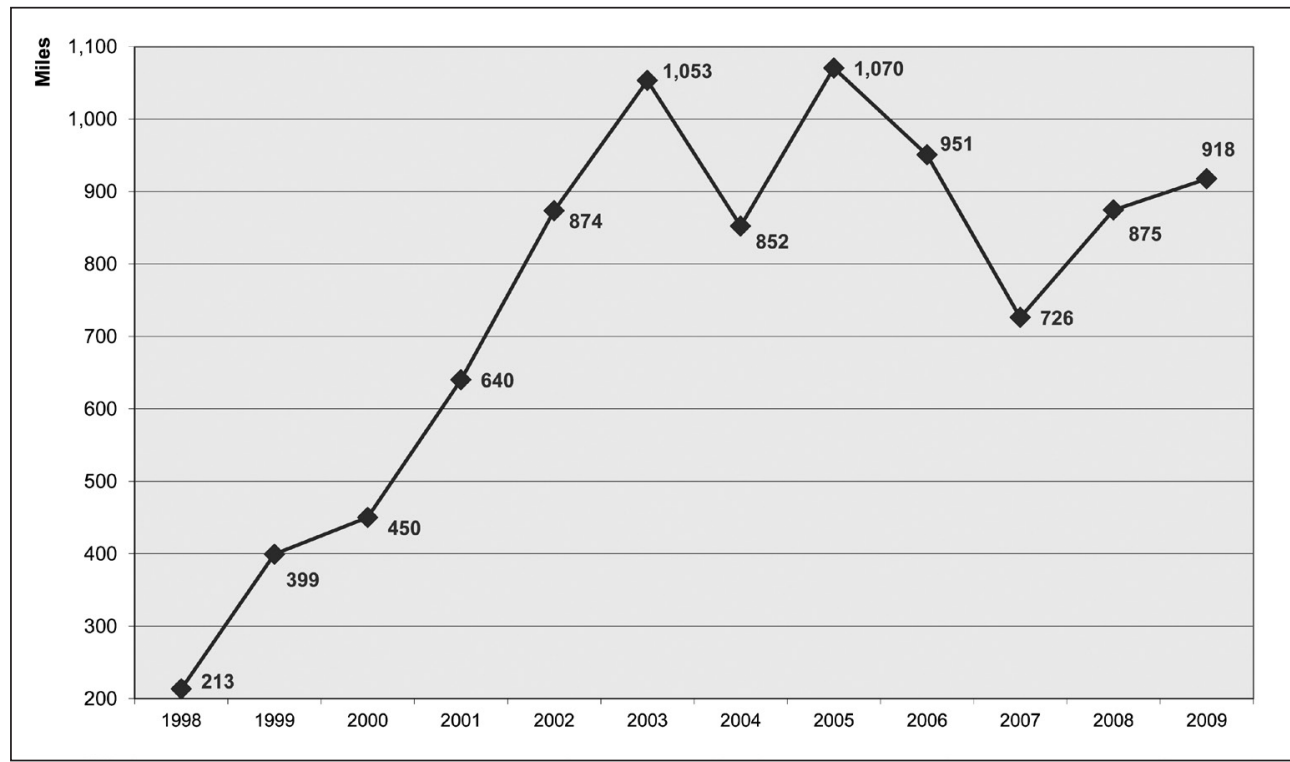

Fuente: Elaboración propia a partir de las estimaciones intercensales, la ePOBa y el Padrón municipal.

El gráfico 2 muestra el mismo perfil desde una perspectiva diferente, la diferencia entre ambas fuentes de información acerca de la población, i.e. la altura entre los puntos negros y grises del gráfico 1.6 Desde el último censo, la diferencia entre ambas cifras de población permanece en el entorno del millón de personas, y muestra una tendencia notablemente estable, que el cambio legislativo que tuvo lugar en la normativa padronal acerca del registro de la población extranjera sin permiso de residencia permanente, y que entró en vigor con efectos a 1 de enero de $2006,{ }^{7}$ no ha sido capaz de cerrar en una magnitud significativa.

En otras palabras, a pesar de que ambas fuentes muestran la misma tendencia de crecimiento, ambas fuentes de población presentan una discrepancia en los niveles relativamente estable desde el último censo, discrepancia que ha demostrado ser extraordinariamente difícil de cerrar en la práctica.

6 El gráfico 2 muestra las discrepancias absolutas, pero el mismo perfil se observa en términos de discrepancias relativas.

7 La Ley de Extranjería de 20 de noviembre de 2003, Ley Orgánica 14/2003. Esta ley introduce la obligación de registrarse de nuevo cada dos años a aquellos extranjeros que, estando ya inscritos, no pertenezcan a la Unión Europea y no dispongan de un permiso de residencia permanente. El objetivo de esta medida es evitar mantener como inscritas en el Padrón Municipal a personas que realmente ya no residen en España, y en consecuencia producen una sobre estimación de la población realmente residente de nuestro país. La ley, cuyos efectos se observaron por vez primera en la Revisión del Padrón a 1 de enero de 2006, tuvo un impacto de cierta magnitud en las cifras provisionales del Padrón de 2006 (INE 2006), pero no en las definitivas, y claramente no ha servido para romper la tendencia. 
Como puede observarse en el gráfico 2, la discrepancia absoluta entre la cifra de la ePOBa y la del Padrón a 1 de enero de 2002 es ligeramente inferior al millón de habitantes (874 mil personas), lo que representa una discrepancia relativa (del Padrón con respecto a la $e P O B a$ ) del $2.13 \%$ (de forma que el Padrón genera una cifra más elevada de población). Debido al desfase temporal entre el Censo de 2001, fechado a 1 de noviembre, y la de las revisiones padronales, fechadas a 1 de enero, la cifra de la $e P O B a$ referida a 1 de enero de 2002 se toma «como si fuera la del Censo de 2001» a efectos de comparación. De hecho, sin este ajuste temporal, la diferencia entre el Censo y el Padrón alcanza exactamente el millón de personas (990.523 habitantes). Sin embargo, esta es una cuestión de detalle que tiene más importancia de la que parece a primera vista y sobre la que volveremos más adelante.

Como veremos posteriormente con cierto detalle, esta divergencia entre fuentes de información es heterogénea a lo largo de las diversas unidades geográficas consideradas en función de determinadas características, tanto observables como no observables. Adicionalmente, estas diferencias crecen conforme nos movemos hacia unidades geográficas más pequeñas. Esto es, existe mayor variabilidad en las discrepancias a nivel provincial (NUTS 3) que a nivel de Comunidad Autónoma (CCAA, NUTS 2).

Por ejemplo, incluso si la discrepancia relativa a nivel nacional es del 2.13\%; a nivel de CCAA el rango de discrepancias va desde el 1.05\% (Castilla y León) al $5.06 \%$ (Illes Balears) ${ }^{8}$ con una desviación típica del $1.3 \%$. A nivel provincial el rango de discrepancias relativas se incrementa desde un valor ligeramente negativo, $-0.35 \%$ (Granada), hasta el $6.54 \%$ (Alicante), con una desviación típica del 1.5\%. A nivel municipal tendremos ocasión de comprobar cómo las discrepancias pueden llegar a ser enormes si las comparamos con estas cifras regionales.

El trabajo se organiza en torno a dos grandes temas. En primer lugar, se comparan con detalle las cifras de población del Censo de 2001 y de la ePOBa de $2002^{9}$ con las del Padrón del mismo año a nivel municipal, una vez las hemos estudiado a nivel regional. El objetivo es poner de manifiesto la enorme heterogeneidad encontrada a pequeña escala, así como mostrar los patrones que explican las discrepancias. En segundo lugar, y tomando una provincia como caso de estudio, tratamos de elaborar estimaciones de población actual a nivel municipal, esto es, poblaciones municipales ancladas en los niveles del Censo de 2001 y que a su vez sean consistentes con las cifras provinciales de la ePOBa proporcionadas por el INE, llamaremos a estas cifras de población Estimaciones de la Población Actual municipal, ePOBa, si bien como se hará explícito posteriormente la metodología es ligeramente diferente de la utilizada por el INE en sus estimaciones.

No cabe duda de que la solución más simple para obtener estas estimaciones de población a nivel municipal, redistribuir la cifra regional de la $e P O B a$ de acuerdo con la estructura relativa derivada del Padrón, proporcionará malos resultados. La razón es muy simple, a pequeña escala las discrepancias no son sólo muy elevadas, sino también enormemente heterogéneas. Documentaremos ampliamente este hecho intentando descubrir los patrones de dicha heterogeneidad y mostrando como la geografía importa enormemente en pequeñas áreas, puesto

8 Esto excluye Ceuta y Melilla, que en conjunto presentan una discrepancia relativa del 5.23\%.

9 Las cifras de las $е P O B a$ a fecha 1 de enero de 2002 coinciden con las estimaciones intercensales de población a la misma fecha, ya que a estos efectos el Censo de 2001 fue trasladado al 1 de enero más cercano. 
que estas discrepancias tienden a agruparse. El análisis de estas discrepancias a diferentes niveles de agregación geográfica forma parte de las secciones 2, 3 y 4.

A continuación abordaremos el problema de construir $e P O B a$ a nivel municipal que sean consistentes con las $e P O B a$ provinciales publicadas por el INE. Puesto que la modelización no ha dado buenos resultados, para dicho ejercicio utilizaremos el método de componentes que, con un cierto desfase, proporciona resultados más que aceptables y es sencillo de implementar en la práctica. Al contrario que la metodología del INE adoptamos aquí un enfoque bottom-up. Este es el contenido de la sección 5. Finalmente una breve sección concluye el trabajo.

\section{CENSO DE 2001, ePOBa Y PADRÓN A NIVEL REGIONAL}

El cuadro 1 ofrece cifras de población a nivel de CCAA (NUTS 2) de acuerdo con las diferentes fuentes contempladas, al mismo tiempo que ofrece las discrepancias absolutas y relativas entre la $e P O B a$ y el Padrón a fecha 1 de enero de 2002. ${ }^{10}$ Un primer patrón interesante

Cuadro 1

POBLACIÓN TOTAL: ESPAÑA Y CCAA (NUTS 2) DEACUERDO CON DIFERENTES FUENTES

\begin{tabular}{|c|c|c|c|c|c|}
\hline \multirow[b]{3}{*}{ España } & \multirow{3}{*}{$\begin{array}{r}\text { Censo } 2001 \\
\begin{array}{r}1^{\circ} \text { Noviembre } \\
40,847,371\end{array}\end{array}$} & \multirow{3}{*}{$\begin{array}{l}\text { ePOBa } 2002 \\
1^{\circ} \text { Enero } \\
40,964,244\end{array}$} & \multirow{3}{*}{$\begin{array}{l}\text { Padrón } 2002 \\
\begin{array}{l}1^{\circ} \text { Enero } \\
41,837,894\end{array}\end{array}$} & \multicolumn{2}{|c|}{$\begin{array}{c}\text { Differencia Padrón } 2002 \\
\text { ePOBa } 2002\end{array}$} \\
\hline & & & & Absoluta & Relativa \\
\hline & & & & 873,650 & $2.13 \%$ \\
\hline 1 Castilla y León & $2,456,474$ & $2,454,546$ & $2,480,369$ & 25,823 & $1.05 \%$ \\
\hline 2 Asturias (Principado de) & $1,062,998$ & $1,061,942$ & $1,073,971$ & 12,029 & $1.13 \%$ \\
\hline 3 Aragón & $1,204,215$ & $1,203,660$ & $1,217,514$ & 13,854 & $1.15 \%$ \\
\hline 4 Castilla-La Mancha & $1,760,516$ & $1,760,162$ & $1,782,038$ & 21,876 & $1.24 \%$ \\
\hline 5 País Vasco & $2,082,587$ & $2,082,258$ & $2,108,281$ & 26,023 & $1.25 \%$ \\
\hline 6 Cantabria & 535,131 & 534,915 & 542,275 & 7,360 & $1.38 \%$ \\
\hline 7 Extremadura & $1,058,503$ & $1,058,148$ & $1,073,050$ & 14,902 & $1.41 \%$ \\
\hline 8 Andalucía & $7,357,558$ & $7,360,469$ & $7,478,432$ & 117,963 & $1.60 \%$ \\
\hline 9 Galicia & $2,695,880$ & $2,693,733$ & $2,737,370$ & 43,637 & $1.62 \%$ \\
\hline 10 Rioja (La) & 276,702 & 276,679 & 281,614 & 4,935 & $1.78 \%$ \\
\hline 11 Madrid (Comunidad de) & $5,423,384$ & $5,426,248$ & $5,527,152$ & 100,904 & $1.86 \%$ \\
\hline 12 Murcia (Región de) & $1,197,646$ & $1,198,606$ & $1,226,993$ & 28,387 & $2.37 \%$ \\
\hline 13 Navarra (Comunidad Foral de) & 555,829 & 555,879 & 569,628 & 13,749 & $2.47 \%$ \\
\hline 14 Cataluña & $6,343,110$ & $6,343,786$ & $6,506,440$ & 162,654 & $2.56 \%$ \\
\hline 15 Canarias & $1,694,477$ & $1,779,169$ & $1,843,755$ & 64,586 & $3.63 \%$ \\
\hline 16 Comunidad Valenciana & $4,162,776$ & $4,163,094$ & $4,326,708$ & 163,614 & $3.93 \%$ \\
\hline 17 Balears (Illes) & 841,669 & 872,836 & 916,968 & 44,132 & $5.06 \%$ \\
\hline 18 Ceuta y Melilla & 137,916 & 138,114 & 145,336 & 7,222 & $5.23 \%$ \\
\hline
\end{tabular}

Nota: La población del Censo va referida al 1 de noviembre de 2001, la ePOBa y el Padrón van referidas al 1 de enero de 2002.

Nota: La estimación Intercensal y la $e P O B a$ de 2002 son coincidentes.

Nota: En negrita el menor valor y en cursiva el máximo valor en el corte transversal.

Fuente: Censo de Población y Viviendas, 2001. Estimaciones de Población Actual. Padrón Municipal, 2002. INE (web).

10 Cómo ya hemos mencionado anteriormente, a efectos de comparación nos centramos en la $e P O B a$ y el Padrón, dado que estas dos fuentes coinciden en su fecha de referencia. 
Cuadro 2

POBLACIÓN TOTAL: ESPAÑA Y PROVINCIAS (NUTS 3) DEACUERDO CON DIFERENTES FUENTES

\begin{tabular}{|c|c|c|c|c|c|}
\hline \multirow[b]{2}{*}{ España } & \multirow{2}{*}{$\begin{array}{r}\text { Censo } 2001 \\
1^{\circ} \text { Noviembre } \\
40,847,371\end{array}$} & \multirow{2}{*}{$\begin{array}{l}\text { ePOBa } 2002 \\
1^{\circ} \text { Enero } \\
40,964,244\end{array}$} & \multirow{2}{*}{$\begin{array}{l}\text { Padrón } 2002 \\
\begin{array}{l}1^{\circ} \text { Enero } \\
41,837,894\end{array}\end{array}$} & \multicolumn{2}{|c|}{$\begin{array}{c}\text { Differencia Padrón } 2002 \\
\text { ePOBa } 2002\end{array}$} \\
\hline & & & & $\begin{array}{l}\text { Absoluta } \\
\qquad 873,650\end{array}$ & $\begin{array}{r}\text { Relativa } \\
2.13 \%\end{array}$ \\
\hline 1 Granada & 821,660 & 821,838 & 818,959 & $-2,879$ & $-0.35 \%$ \\
\hline 2 Huelva & 462,579 & 462,689 & 464,934 & 2,245 & $0.49 \%$ \\
\hline 3 Salamanca & 345,609 & 345,324 & 347,120 & 1,796 & $0.52 \%$ \\
\hline 4 Jaén & 643,820 & 643,889 & 647,387 & 3,498 & $0.54 \%$ \\
\hline 5 Valladolid & 498,094 & 498,043 & 501,157 & 3,114 & $0.63 \%$ \\
\hline 6 Cuenca & 200,346 & 200,241 & 201,614 & 1,373 & $0.69 \%$ \\
\hline 7 Soria & 90,717 & 90,669 & 91,487 & 818 & $0.90 \%$ \\
\hline 8 Zamora & 199,090 & 198,840 & 200,678 & 1,838 & $0.92 \%$ \\
\hline 9 Toledo & 541,379 & 541,254 & 546,538 & 5,284 & $0.98 \%$ \\
\hline 10 Vizcaya & $1,122,637$ & $1,122,236$ & $1,133,444$ & 11,208 & $1.00 \%$ \\
\hline 11 Segovia & 147,694 & 147,628 & 149,286 & 1,658 & $1.12 \%$ \\
\hline 12 Zaragoza & 861,855 & 861,518 & 871,209 & 9,691 & $1.12 \%$ \\
\hline 13 Burgos & 348,934 & 348,786 & 352,723 & 3,937 & $1.13 \%$ \\
\hline 14 Ávila & 163,442 & 163,294 & 165,138 & 1,844 & $1.13 \%$ \\
\hline 15 Asturias & $1,062,998$ & $1,061,942$ & $1,073,971$ & 12,029 & $1.13 \%$ \\
\hline 16 Ciudad Real & 478,957 & 478,839 & 484,338 & 5,499 & $1.15 \%$ \\
\hline 17 Teruel & 135,858 & 135,749 & 137,342 & 1,593 & $1.17 \%$ \\
\hline 18 Córdoba & 761,657 & 761,861 & 771,131 & 9,270 & $1.22 \%$ \\
\hline 19 Badajoz & 654,882 & 654,711 & 662,808 & 8,097 & $1.24 \%$ \\
\hline 20 Huesca & 206,502 & 206,393 & 208,963 & 2,570 & $1.25 \%$ \\
\hline 21 Palencia & 174,143 & 173,949 & 176,125 & 2,176 & $1.25 \%$ \\
\hline 22 Lugo & 357,648 & 357,050 & 361,782 & 4,732 & $1.33 \%$ \\
\hline 23 Cantabria & 535,131 & 534,915 & 542,275 & 7,360 & $1.38 \%$ \\
\hline 24 Guipúzcoa & 673,563 & 673,596 & 682,977 & 9,381 & $1.39 \%$ \\
\hline 25 Coruña $(A)$ & $1,096,027$ & $1,095,177$ & $1,111,886$ & 16,709 & $1.53 \%$ \\
\hline 26 Guadalajara & 174,999 & 174,998 & 177,761 & 2,763 & $1.58 \%$ \\
\hline 27 Cáceres & 403,621 & 403,437 & 410,242 & 6,805 & $1.69 \%$ \\
\hline 28 Ourense & 338,446 & 337,968 & 343,768 & 5,800 & $1.72 \%$ \\
\hline 29 Sevilla & $1,727,603$ & $1,728,241$ & $1,758,720$ & 30,479 & $1.76 \%$ \\
\hline 30 Almería & 536,731 & 537,013 & 546,498 & 9,485 & $1.77 \%$ \\
\hline 31 León & 488,751 & 488,013 & 496,655 & 8,642 & $1.77 \%$ \\
\hline 32 Rioja (La) & 276,702 & 276,679 & 281,614 & 4,935 & $1.78 \%$ \\
\hline 33 Pontevedra & 903,759 & 903,538 & 919,934 & 16,396 & $1.81 \%$ \\
\hline 34 Madrid & $5,423,384$ & $5,426,248$ & $5,527,152$ & 100,904 & $1.86 \%$ \\
\hline 35 Álava & 286,387 & 286,426 & 291,860 & 5,434 & $1.90 \%$ \\
\hline 36 Albacete & 364,835 & 364,830 & 371,787 & 6,957 & $1.91 \%$ \\
\hline 37 Barcelona & $4,805,927$ & $4,806,534$ & $4,906,117$ & 99,583 & $2.07 \%$ \\
\hline 38 Cádiz & $1,116,491$ & $1,117,190$ & $1,140,793$ & 23,603 & $2.11 \%$ \\
\hline 39 Valencia/València & $2,216,285$ & $2,216,191$ & $2,267,503$ & 51,312 & $2.32 \%$ \\
\hline 40 Murcia & $1,197,646$ & $1,198,606$ & $1,226,993$ & 28,387 & $2.37 \%$ \\
\hline 41 Navarra & 555,829 & 555,879 & 569,628 & 13,749 & $2.47 \%$ \\
\hline 42 Lleida & 362,206 & 362,080 & 371,055 & 8,975 & $2.48 \%$ \\
\hline 43 Palmas (Las) & 887,676 & 925,160 & 951,037 & 25,877 & $2.80 \%$ \\
\hline 44 Málaga & $1,287,017$ & $1,287,748$ & $1,330,010$ & 42,262 & $3.28 \%$ \\
\hline 45 Castellón/Castelló & 484,566 & 484,585 & 501,237 & 16,652 & $3.44 \%$ \\
\hline 46 Tarragona & 609,673 & 609,787 & 631,156 & 21,369 & $3.50 \%$ \\
\hline 47 Melilla & 66,411 & 66,529 & 69,184 & 2,655 & $3.99 \%$ \\
\hline 48 Santa Cruz de Tenerife & 806,801 & 854,009 & 892,718 & 38,709 & $4.53 \%$ \\
\hline 49 Balears (Illes) & 841,669 & 872,836 & 916,968 & 44,132 & $5.06 \%$ \\
\hline 50 Girona & 565,304 & 565,385 & 598,112 & 32,727 & $5.79 \%$ \\
\hline 51 Ceuta & 71,505 & 71,585 & 76,152 & 4,567 & $6.38 \%$ \\
\hline 52 Alicante/Alacant & $1,461,925$ & $1,462,318$ & $1,557,968$ & 95,650 & $6.54 \%$ \\
\hline
\end{tabular}

Nota: La población del Censo va referida al 1 de noviembre de 2001, la ePOBa y el Padrón van referidas al 1 de enero de 2002 .

Nota: La estimación Intercensal y la $e P O B a$ de 2002 son coincidentes.

Nota: En negrita el menor valor y en cursiva el máximo valor en el corte transversal.

Fuente: Censo de Población y Viviendas, 2001. Estimaciones de Población Actual. Padrón Municipal, 2002. INE (web). 
emerge, 7 regiones muestran una discrepancia por encima de la media global (la cifra nacional, 2.13\%), pero todas ellas, con la excepción de Navarra, son islas o están situadas en la costa mediterránea. Observamos discrepancias por encima del 5\% en un par de casos (Illes Balears y Ceuta y Melilla), y estas no son en absoluto cifras insignificantes.

El cuadro 2 muestra la misma información pero a nivel provincial (NUTS 3). Como ya hemos mencionado, el rango de las discrepancias se incrementa de forma considerable. Esto es cierto a ambos lados de la distribución. No sólo observamos un caso en el que el Padrón proporciona una cifra de población inferior a la del Censo (Granada), pero también 4 provincias con discrepancias positivas por encima del 5\% (Illes Balears, Girona, Ceuta y Alicante), todas ellas en la costa mediterránea. En un mundo linealmente homogéneo las discrepancias deberían ser constantes a lo largo de los diferentes territorios, lo que en vista de los cuadros 1 y 2 podemos estar seguros de que no es el caso.

La geografía de las discrepancias es marcada y claramente visible en el mapa 1; las islas, las ciudades autónomas africanas (Ceuta y Melilla) y la costa mediterránea parecen ser los lugares donde la población es más difícil de contar.

Además, como es natural, las discrepancias absolutas y el nivel de población están altamente correlacionadas (con un coeficiente de correlación de 0.86), sin embargo las discrepancias relativas parecen no guardar relación con el stock de población de la provincia (siendo la correlación tan sólo de 0.08). Puesto que estas discrepancias no son constantes, sino que por el contrario muestran una elevada variabilidad, otros factores deben ser los

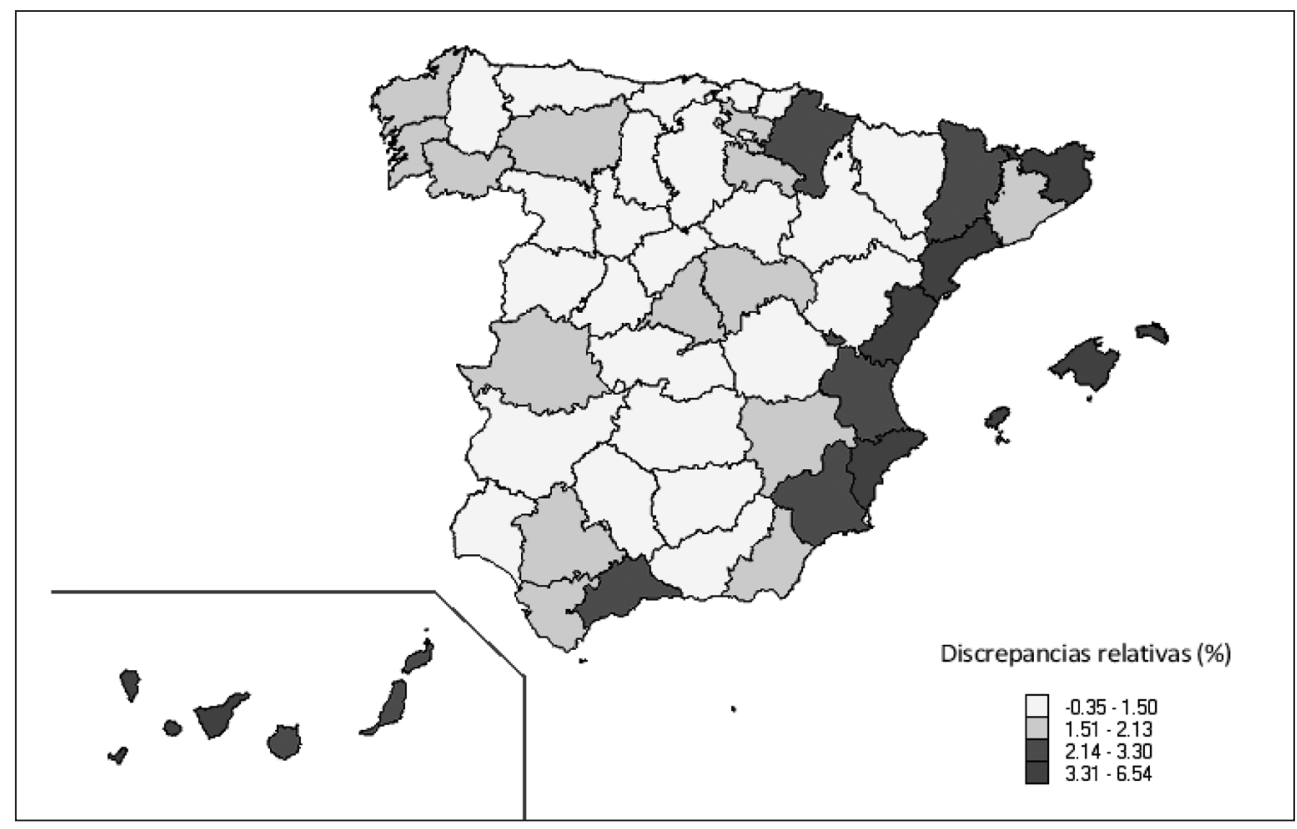

Fuente: Elaboración propia a partir de la $e P O B a$ y el Padrón municipal. 
responsables de las mismas, desvelarlas a nivel municipal es parte de los objetivos de este trabajo.

\section{CENSO DE 2001 VERSUS ePOBa DE 2002}

Antes de examinar las discrepancias a nivel municipal, es conveniente examinar a nivel provincial, y con algo más de cercanía, las cifras del Censo de 2001, fechadas a 1 de noviembre, y las de la $e P O B a$, fechadas a 1 de enero de 2002, mostradas en el cuadro 2.

El cuadro 3 ofrece la información relevante. El lapso temporal entre ambas cifras es de sólo dos meses, por lo que las discrepancias son muy pequeñas. Las cifras agregadas de la ePOBa proporcionan, lógicamente, un valor superior a las del censo estimada en 116,873 personas más, lo que representa un crecimiento en esos dos meses del 0.29\%. En todas las provincias las discrepancias relativas son inferiores al $0.2 \%$ excepto en tres casos curiosos, las islas. Las diferencias en las tres provincias insulares están fuera de cualquier tendencia y muy por encima del $3 \%$ en todos los casos, lo que representa un crecimiento de la población poco creíble. En concreto, la discrepancia relativa es de un $3.70 \%$ en Illes Balears, de un 4.22\% en Las Palmas y de un 5.85\% en Santa Cruz de Tenerife. Estas cifras son enormes si las tomamos como el crecimiento de la población en estas provincias en tan solo dos meses. De hecho, estas tres provincias justifican 115,859 personas del crecimiento agregado de la población, por lo que eliminarlas reduciría la discrepancia entre las cifras del censo y las de la $e P O B a$ a una cifra insignificante, 1,014 personas. Por tanto, en la práctica casi todas las discrepancias agregadas pueden ser atribuidas a las provincias insulares, o visto desde otra perspectiva, todo el crecimiento de la población española entre noviembre y diciembre de 2001 se debe al crecimiento de la población en las provincias insulares.

Una visión más realista de estas discrepancias es considerar que las islas tienen sus propios problemas de medida en relación al recuento de la población. Esta es efectivamente la razón de estas peculiares diferencias.

El propio INE ( in fecha, p.-6) indica, al comentar la población de partida en la $e P O B a$ que las cifras del Censo de 2001 se ajustaron, para cada provincia, con el crecimiento vegetativo durante los meses de noviembre y diciembre de 2001, mientras que «...en lo que se refiere a los movimientos migratorios producidos en esos dos meses, la práctica totalidad de los mismos se consideran recogidos en la propia operación censal, dada la duración del trabajo de campo de la misma, y por tanto reflejados en el propio Censo.» (INE, 2009b, p.-7). Es decir, no se realizó ningún ajuste por migraciones para llevar las poblaciones del censo al punto de partida para las estimaciones de la $e P O B a$, el 1 de enero de 2002.

Estos ajustes se muestran en el cuadro 4. Aunque la consideración del crecimiento vegetativo no hacer coincidir exactamente las cifras ajustadas del Censo con las de la $e P O B a$, si reduce enormemente las diferencias. Quizá ello es debido a que el ajuste se realizó con cifras no definitivas del Censo o del Movimiento Natural de la Población, ya que aunque la $e P O B a$ se hicieron públicas en julio de 2007 se venían elaborando por parte del INE de forma interna, tanto a efectos de suministrar con regularidad las cifras de población que demandan otras operaciones estadísticas (encuestas, contabilidad nacional, indicadores demográ- 
Cuadro 3

CENSO DE 2001, $1^{\circ}$ NOVIEMBRE, Y ePOBa de 2002, $1^{\circ}$ ENERO

\begin{tabular}{|c|c|c|c|c|}
\hline & \multirow{2}{*}{$\begin{array}{l}\text { Censo } 2001 \\
1^{\circ} \text { Noviembre }\end{array}$} & \multirow{2}{*}{$\begin{array}{c}\text { ePOBa } 2002 \\
1^{\circ} \text { Enero }\end{array}$} & \multicolumn{2}{|c|}{$\begin{array}{c}\text { Differencia ePOBa } 2002 \\
\text { Censo } 2001\end{array}$} \\
\hline & & & Absoluta & Relativa \\
\hline España & $40,847,371$ & $40,964,244$ & 116,873 & $0.29 \%$ \\
\hline 1 Lugo & 357,648 & 357,050 & -598 & $-0.17 \%$ \\
\hline 2 León & 488,751 & 488,013 & -738 & $-0.15 \%$ \\
\hline 3 Ourense & 338,446 & 337,968 & -478 & $-0.14 \%$ \\
\hline 4 Zamora & 199,090 & 198,840 & -250 & $-0.13 \%$ \\
\hline 5 Palencia & 174,143 & 173,949 & -194 & $-0.11 \%$ \\
\hline 6 Asturias & $1,062,998$ & $1,061,942$ & $-1,056$ & $-0.10 \%$ \\
\hline 7 Ávila & 163,442 & 163,294 & -148 & $-0.09 \%$ \\
\hline 8 Salamanca & 345,609 & 345,324 & -285 & $-0.08 \%$ \\
\hline 9 Teruel & 135,858 & 135,749 & -109 & $-0.08 \%$ \\
\hline 10 Coruña $(A)$ & $1,096,027$ & $1,095,177$ & -850 & $-0.08 \%$ \\
\hline 11 Soria & 90,717 & 90,669 & -48 & $-0.05 \%$ \\
\hline 12 Huesca & 206,502 & 206,393 & -109 & $-0.05 \%$ \\
\hline 13 Cuenca & 200,346 & 200,241 & -105 & $-0.05 \%$ \\
\hline 14 Cáceres & 403,621 & 403,437 & -184 & $-0.05 \%$ \\
\hline 15 Segovia & 147,694 & 147,628 & -66 & $-0.04 \%$ \\
\hline 16 Burgos & 348,934 & 348,786 & -148 & $-0.04 \%$ \\
\hline 17 Cantabria & 535,131 & 534,915 & -216 & $-0.04 \%$ \\
\hline 18 Zaragoza & 861,855 & 861,518 & -337 & $-0.04 \%$ \\
\hline 19 Vizcaya & $1,122,637$ & $1,122,236$ & -401 & $-0.04 \%$ \\
\hline 20 Lleida & 362,206 & 362,080 & -126 & $-0.03 \%$ \\
\hline 21 Badajoz & 654,882 & 654,711 & -171 & $-0.03 \%$ \\
\hline 22 Ciudad Real & 478,957 & 478,839 & -118 & $-0.02 \%$ \\
\hline 23 Pontevedra & 903,759 & 903,538 & -221 & $-0.02 \%$ \\
\hline 24 Toledo & 541,379 & 541,254 & -125 & $-0.02 \%$ \\
\hline 25 Valladolid & 498,094 & 498,043 & -51 & $-0.01 \%$ \\
\hline 26 Rioja (La) & 276,702 & 276,679 & -23 & $-0.01 \%$ \\
\hline 27 Valencia/València & $2,216,285$ & $2,216,191$ & -94 & $0.00 \%$ \\
\hline 28 Albacete & 364,835 & 364,830 & -5 & $0.00 \%$ \\
\hline 29 Guadalajara & 174,999 & 174,998 & -1 & $0.00 \%$ \\
\hline 30 Castellón/Castelló & 484,566 & 484,585 & 19 & $0.00 \%$ \\
\hline 31 Guipúzcoa & 673,563 & 673,596 & 33 & $0.00 \%$ \\
\hline 32 Navarra & 555,829 & 555,879 & 50 & $0.01 \%$ \\
\hline 33 Jaén & 643,820 & 643,889 & 69 & $0.01 \%$ \\
\hline 34 Barcelona & $4,805,927$ & $4,806,534$ & 607 & $0.01 \%$ \\
\hline 35 Álava & 286,387 & 286,426 & 39 & $0.01 \%$ \\
\hline 36 Girona & 565,304 & 565,385 & 81 & $0.01 \%$ \\
\hline 37 Tarragona & 609,673 & 609,787 & 114 & $0.02 \%$ \\
\hline 38 Granada & 821,660 & 821,838 & 178 & $0.02 \%$ \\
\hline 39 Huelva & 462,579 & 462,689 & 110 & $0.02 \%$ \\
\hline 40 Córdoba & 761,657 & 761,861 & 204 & $0.03 \%$ \\
\hline 41 Alicante/Alacant & $1,461,925$ & $1,462,318$ & 393 & $0.03 \%$ \\
\hline 42 Sevilla & $1,727,603$ & $1,728,241$ & 638 & $0.04 \%$ \\
\hline 43 Almería & 536,731 & 537,013 & 282 & $0.05 \%$ \\
\hline 44 Madrid & $5,423,384$ & $5,426,248$ & 2,864 & $0.05 \%$ \\
\hline 45 Málaga & $1,287,017$ & $1,287,748$ & 731 & $0.06 \%$ \\
\hline 46 Cádiz & $1,116,491$ & $1,117,190$ & 699 & $0.06 \%$ \\
\hline 47 Murcia & $1,197,646$ & $1,198,606$ & 960 & $0.08 \%$ \\
\hline 48 Ceuta & 71,505 & 71,585 & 80 & $0.11 \%$ \\
\hline 49 Melilla & 66,411 & 66,529 & 118 & $0.18 \%$ \\
\hline 50 Balears (Illes) & 841,669 & 872,836 & 31,167 & $3.70 \%$ \\
\hline 51 Palmas (Las) & 887,676 & 925,160 & 37,484 & $4.22 \%$ \\
\hline 52 Santa Cruz de Tenerife & 806,801 & 854,009 & 47,208 & $5.85 \%$ \\
\hline
\end{tabular}

Nota: La población del Censo va referida al 1 de noviembre de 2001, la ePOBa y el Padrón van referidas al 1 de enero de 2002.

Nota: La estimación Intercensal y la $e P O B a$ de 2002 son coincidentes.

Nota: En negrita el menor valor y en cursiva el máximo valor en el corte transversal.

Fuente: Censo de Población y Viviendas, 2001. Estimaciones de Población Actual. Padrón Municipal, 2002. INE (web). 
Cuadro 4

CENSO DE 2001, $1^{\circ}$ NOVIEMBRE. DEFUNCIONES Y NACIMIENTOS, NOVIEMBRE Y DICIEMBRE 2001, CENSO DE 2001 AJUSTADO POR CRECIMIENTO VEGETATIVO A $1^{\circ}$ ENERO DE 2002 Y ePOBa de 2002, $1^{\circ}$ ENERO

\begin{tabular}{|c|c|c|c|c|c|}
\hline & $\begin{array}{c}\text { Censo } 2001 \\
1^{\circ} \text { Noviembre }\end{array}$ & $\begin{array}{l}\text { Crecimiento } \\
\text { vegetativo: } \\
\text { Nov.-Dic. } 2001\end{array}$ & $\begin{array}{c}\text { Censo } 2001 \\
\text { ajustado } \\
1^{\circ} \text { Enero } 2002\end{array}$ & $\begin{array}{c}\text { ePOBa } 2002 \\
1^{\circ} \text { Enero }\end{array}$ & $\begin{array}{c}\text { Diferencia } \\
\text { ePOBa } 2002 \\
\text { Censo ajustado }\end{array}$ \\
\hline España & $40,847,371$ & 2,805 & $40,850,176$ & $40,964,244$ & 114,068 \\
\hline 1 Álava & 286,387 & -7 & 286,380 & 286,426 & 46 \\
\hline 2 Albacete & 364,835 & -11 & 364,824 & 364,830 & 6 \\
\hline 3 Alicante/Alacant & $1,461,925$ & 348 & $1,462,273$ & $1,462,318$ & 45 \\
\hline 4 Almería & 536,731 & 332 & 537,063 & 537,013 & -50 \\
\hline 5 Ávila & 163,442 & -169 & 163,273 & 163,294 & 21 \\
\hline 6 Badajoz & 654,882 & -140 & 654,742 & 654,711 & -31 \\
\hline 7 Balears (Illes) & 841,669 & 393 & 842,062 & 872,836 & 30,774 \\
\hline 8 Barcelona & $4,805,927$ & 736 & $4,806,663$ & $4,806,534$ & -129 \\
\hline 9 Burgos & 348,934 & -157 & 348,777 & 348,786 & 9 \\
\hline 10 Cáceres & 403,621 & -209 & 403,412 & 403,437 & 25 \\
\hline 11 Cádiz & $1,116,491$ & 726 & $1,117,217$ & $1,117,190$ & -27 \\
\hline 12 Castellón/Castelló & 484,566 & -14 & 484,552 & 484,585 & 33 \\
\hline 13 Ciudad Real & 478,957 & -114 & 478,843 & 478,839 & -4 \\
\hline 14 Córdoba & 761,657 & 143 & 761,800 & 761,861 & 61 \\
\hline 15 Coruña $(A)$ & $1,096,027$ & -759 & $1,095,268$ & $1,095,177$ & -91 \\
\hline 16 Cuenca & 200,346 & -134 & 200,212 & 200,241 & 29 \\
\hline 17 Girona & 565,304 & 55 & 565,359 & 565,385 & 26 \\
\hline 18 Granada & 821,660 & 201 & 821,861 & 821,838 & -23 \\
\hline 19 Guadalajara & 174,999 & -29 & 174,970 & 174,998 & 28 \\
\hline 20 Guipúzcoa & 673,563 & -22 & 673,541 & 673,596 & 55 \\
\hline 21 Huelva & 462,579 & 105 & 462,684 & 462,689 & 5 \\
\hline 22 Huesca & 206,502 & -122 & 206,380 & 206,393 & 13 \\
\hline 23 Jaén & 643,820 & 20 & 643,840 & 643,889 & 49 \\
\hline 24 León & 488,751 & -519 & 488,232 & 488,013 & -219 \\
\hline 25 Lleida & 362,206 & -143 & 362,063 & 362,080 & 17 \\
\hline 26 Rioja (La) & 276,702 & -32 & 276,670 & 276,679 & 9 \\
\hline 27 Lugo & 357,648 & -644 & 357,004 & 357,050 & 46 \\
\hline 28 Madrid & $5,423,384$ & 2,866 & $5,426,250$ & $5,426,248$ & -2 \\
\hline 29 Málaga & $1,287,017$ & 625 & $1,287,642$ & $1,287,748$ & 106 \\
\hline 30 Murcia & $1,197,646$ & 955 & $1,198,601$ & $1,198,606$ & 5 \\
\hline 31 Navarra & 555,829 & 52 & 555,881 & 555,879 & -2 \\
\hline 32 Ourense & 338,446 & -507 & 337,939 & 337,968 & 29 \\
\hline 33 Asturias & $1,062,998$ & $-1,052$ & $1,061,946$ & $1,061,942$ & -4 \\
\hline 34 Palencia & 174,143 & -189 & 173,954 & 173,949 & -5 \\
\hline 35 Palmas (Las) & 887,676 & 859 & 888,535 & 925,160 & 36,625 \\
\hline 36 Pontevedra & 903,759 & -241 & 903,518 & 903,538 & 20 \\
\hline 37 Salamanca & 345,609 & -349 & 345,260 & 345,324 & 64 \\
\hline 38 Santa Cruz de Tenerife & 806,801 & 530 & 807,331 & 854,009 & 46,678 \\
\hline 39 Cantabria & 535,131 & -220 & 534,911 & 534,915 & 4 \\
\hline 40 Segovia & 147,694 & -78 & 147,616 & 147,628 & 12 \\
\hline 41 Sevilla & $1,727,603$ & 759 & $1,728,362$ & $1,728,241$ & -121 \\
\hline 42 Soria & 90,717 & -73 & 90,644 & 90,669 & 25 \\
\hline 43 Tarragona & 609,673 & 33 & 609,706 & 609,787 & 81 \\
\hline 44 Teruel & 135,858 & -127 & 135,731 & 135,749 & 18 \\
\hline 45 Toledo & 541,379 & -63 & 541,316 & 541,254 & -62 \\
\hline 46 Valencia/València & $2,216,285$ & -10 & $2,216,275$ & $2,216,191$ & -84 \\
\hline 47 Valladolid & 498,094 & -131 & 497,963 & 498,043 & 80 \\
\hline 48 Vizcaya & $1,122,637$ & -303 & $1,122,334$ & $1,122,236$ & -98 \\
\hline 49 Zamora & 199,090 & -259 & 198,831 & 198,840 & 9 \\
\hline 50 Zaragoza & 861,855 & -303 & 861,552 & 861,518 & -34 \\
\hline 51 Ceuta & 71,505 & 78 & 71,583 & 71,585 & 2 \\
\hline 52 Melilla & 66,411 & 119 & 66,530 & 66,529 & -1 \\
\hline
\end{tabular}

Nota: La población del Censo va referida al 1 de noviembre de 2001, la ePOBa y el Padrón van referidas al 1 de enero de 2002.

Nota: La estimación Intercensal y la $e P O B a$ de 2002 son coincidentes.

Nota: En negrita el menor valor y en cursiva el máximo valor en el corte transversal.

Fuente: Censo de Población y Viviendas, 2001. Estimaciones de Población Actual. Padrón Municipal, 2002. INE (web). 
ficos,...), como para el suministro de cifras de población a Eurostat y otros organismos internacionales (Naciones Unidas, OCDE, Fondo Monetario Internacional,...). ${ }^{11}$

Los tres casos llamativos son de nuevo las provincias insulares. Pero el INE nos ofrece la explicación: «...se rectificaron las poblaciones censales de las tres provincias insulares, sumándoles unos ciento catorce mil habitantes, de los cuales, aproximadamente treinta y un mil correspondieron a Baleares y treinta y siete mil y cuarenta y siete mil, respectivamente, a Las Palmas y Santa Cruz de Tenerife. Dicha rectificación se llevó a cabo en vista del déficit de cobertura de hojas padronales que la operación de campo del Censo de 2001 tuvo en estas provincias.» (INE, 2009b, p.-7). Estas cifras coinciden exactamente con las del cuadro 4. ${ }^{12}$ Se deduce de ello que las poblaciones del Censo de 2001 de estas provincias están subestimadas. Aún así, llama la atención que este «...déficit de cobertura de hojas padronales...» sea en los 3 casos del orden de magnitud de la mitad de la diferencia entre las cifras del Padrón de 2002 y del Censo de 2001 (cuadro 2).

En lo que respecta a nuestro ejercicio, esto significa que, con excepción de las provincias insulares, podemos ajustar las poblaciones municipales del Censo de 2001 simplemente a partir del crecimiento vegetativo durante los meses de noviembre y diciembre de dicho año, distribuir la escasa diferencia mostrada en el cuadro 4 según un criterio de proporcionalidad, y tomar las poblaciones resultantes, cuya agregación será consistente con la cifra de $e P O B a$ provincial del INE, como cifra de partida para elaborar $e P O B a$ a nivel municipal mediante el método de componentes.

\section{HETEROGENEIDAD EN LAS CIFRAS DE POBLACIÓN MUNICIPAL SEGÚN DIFERENTES FUENTES}

A continuación realizamos el mismo ejercicio de comparación de fuentes estadísticas de población que el realizado en la sección 2 pero a nivel municipal para una provincia concreta. Como caso de estudio tomamos Alicante, que muestra las mayores discrepancias poblacionales entre la $e P O B a$ y el Padrón en 2002, un 6.54\%, algo más de 3 veces el valor para el agregado nacional, $2.13 \%$ (cuadro 2).

Para la estimación de la $e P O B a$ a nivel municipal a 1 de enero de 2002 debemos estimar el crecimiento vegetativo de cada municipio durante los meses de noviembre y diciembre de 2001. El INE no ofrece esta información ya elaborada en su web a nivel municipal y según los distintos meses del año. Este nivel de detalle está disponible para años completos, mientras que a nivel mensual sólo se ofrece dicha información para la capital de provincia y los municipios con población mayor de 50.000 habitantes. Ello tanto para defunciones como para nacimientos. La utilización de los microdatos del Movimiento Natural de la Población

11 En efecto, las cifras que ofrece Eurostat (http://epp.eurostat.ec.europa.eu/) como población de España coinciden con las estimaciones intercensales, primero, y la ePOBa a partir de 2002. Esto genera una situación dual, a efectos internacionales nuestra población oficial es una, las poblaciones de Eurostat son las que determinan en muchas ocasiones el peso político de cada país dentro de la Unión Europea (EU), mientras que a efectos nacionales nuestra población oficial, en un sentido legal, es otra, la del Padrón, y esta es la que juega un papel determinante en las cuestiones de reparto internas.

12 La metodología actualmente disponible en la web, http://www.ine.es/daco/daco43/epoba/metodo.pdf ofrece cifras incorrectas del ajuste, ya que atribuye «...cuarenta mil... a Baleares y treinta mil y cuarenta mil, respectivamente, a Santa Cruz de Tenerife y Las Palmas...» (INE sin fecha, p.-6) (consultado el 19/4/2010). 
(MNP) permite afinar algo más la estimación, ya que ofrece los códigos del municipio de residencia (del fallecido o de la madre del recién nacido) siempre que este tenga más de 10.000 habitantes, lo que en la práctica representa identificar con exactitud más del $80 \%$ de los casos. Para los municipios con población inferior se reparten, proporcionalmente a su población, las defunciones y los nacimientos que no se han atribuido a un municipio concreto. Igualmente, la discrepancia entre los datos censales ajustados por el crecimiento vegetativo y la cifra de $e P O B a$ a fecha de 1 de enero de 2002 para Alicante (45 habitantes, cuadro 4) se reparte proporcionalmente entre todos los municipios. Este proceso proporciona una estimación de las poblaciones municipales consistentes con la $e P O B a$ a esta fecha de referencia y que puede tomarse como punto de partida para su extensión a fechas posteriores.

La comparación de la ePOBa a nivel municipal, elaborada como acabamos de exponer en el párrafo precedente, con las cifras del Padrón de 2002 indica que el rango de discrepancias relativas se incrementa de forma notable cuando descendemos a nivel municipal. A esta escala no es infrecuente encontrar discrepancias negativas, alrededor del $30 \%$ de las observaciones, pero también enormes discrepancias positivas, en 6 casos estas discrepancias relativas exceden el $20 \%$ y en 3 exceden el $40 \%$. Como consecuencia observamos una enorme variabilidad, la desviación estándar es del 10.1\%, más de 6 veces la desviación estándar observada a nivel provincial.

\section{Gráfico 3}

DISCREPANCIAS RELATIVAS: ALICANTE

PADRÓN VERSUS EPOBa, 1 DE ENERO DE 2002

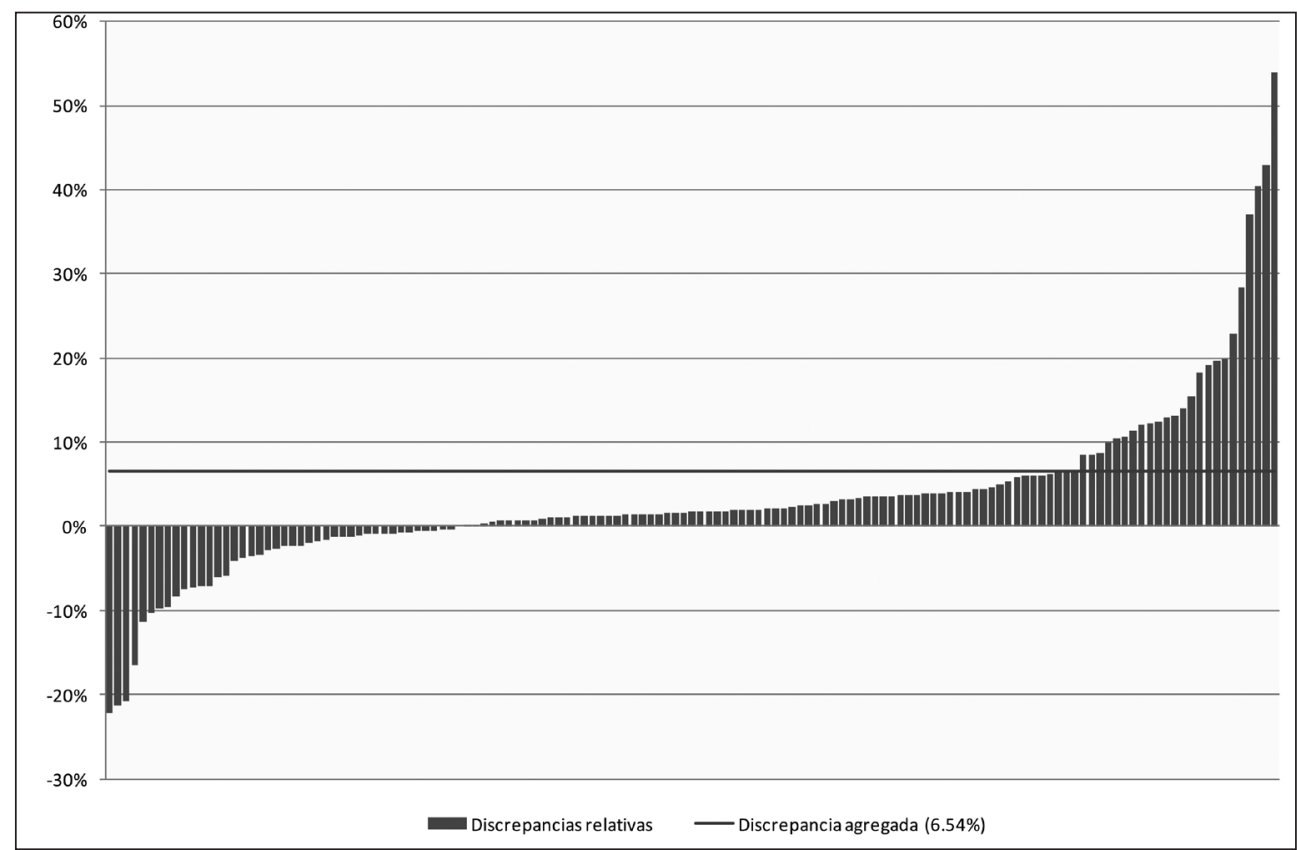

Fuente: Elaboración propia a partir de la $e P O B a$, el Padrón municipal 2002, y los microdatos del Movimiento Natural de la Población, INE. 
Mapa 2

DISCREPANCIAS RELATIVAS: ALICANTE

PADRÓN VERSUS ePOBa, 1 DE ENERO DE 2002

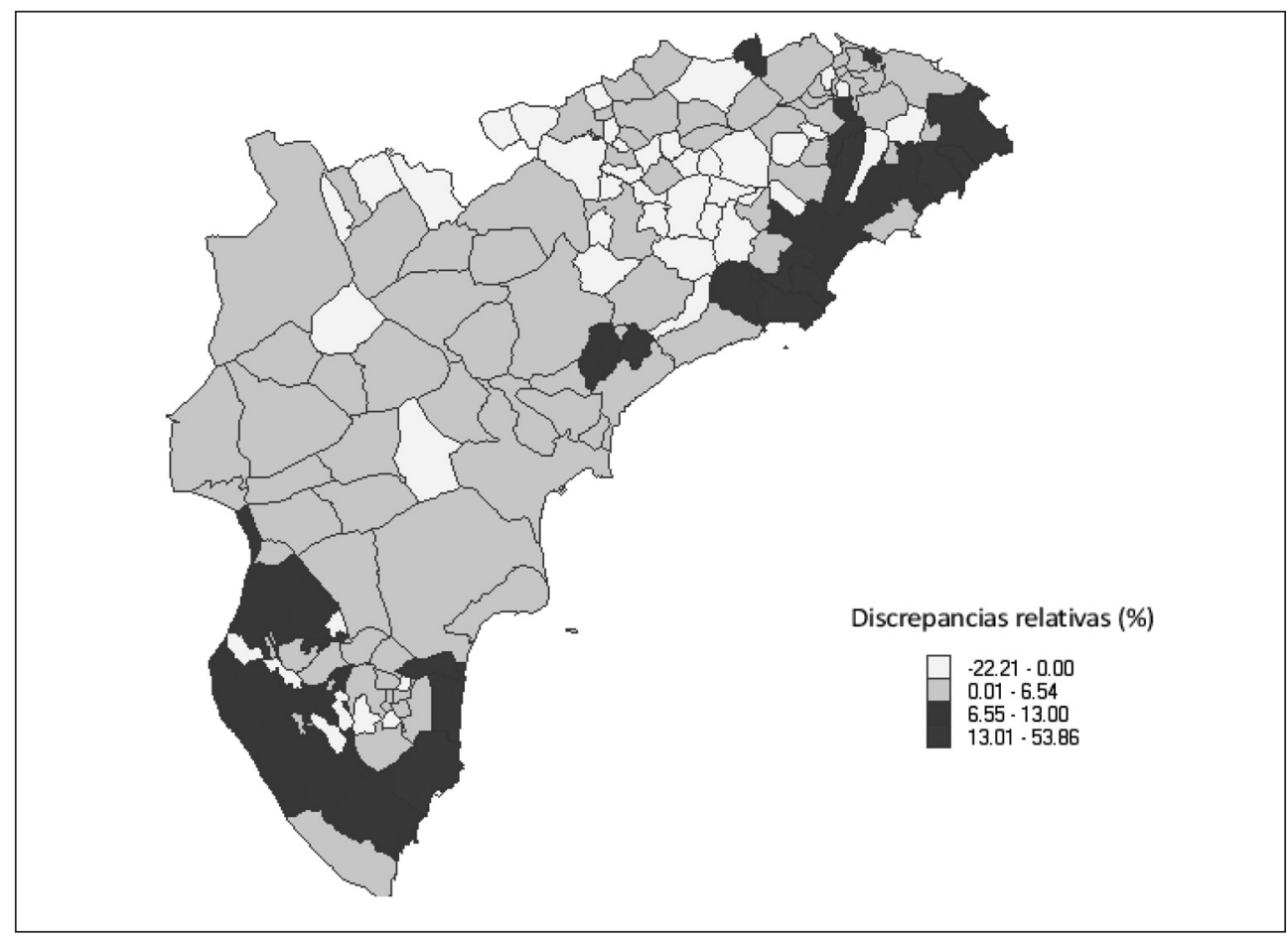

Fuente: Elaboración propia a partir de la $e P O B a$ y el Padrón municipal.

El gráfico 3 ofrece el perfil de estas discrepancias ordenadas de menor a mayor y permite observar claramente como en un gran número de casos el Padrón proporciona una cifra de población inferior a la del censo, así pues a pequeña escala no es cierto que el Padrón sobreestime las cifras de población. Para 22 municipios (alrededor de un 15\% del total) las discrepancias relativas se sitúan en el intervalo $[-1 \%,+1 \%]$, lo que resulta despreciable, pero un gran número de pequeñas áreas muestran grandes discrepancias a ambos lados de la distribución, la modelización de estas discrepancias resulta desafiante, ya que no parece que estas sean aleatorias.

$\mathrm{Al}$ igual que a nivel provincial resulta de interés examinar la geografía municipal de las discrepancias relativas, lo que se muestran en el mapa 2. Se observa con claridad que las mayores discrepancias tienden a localizarse en la costa, mientras que discrepancias pequeñas y negativas se sitúan en el interior. Si este patrón es debido a peculiaridades relacionadas con la geografía física o a características demográficas de los municipios en cuestión es algo que exploraremos de forma tentativa a continuación. Lo que sí es evidente a partir de la inspección del mapa 2 es que estas discrepancias tienen a agruparse en el espacio, de forma que las relaciones de vecindad, además de otras características geográficas, son importantes. 
lidad. Además estas características tienden a estar localizadas geográficamente, un municipio costero siempre tiene, al menos, un vecino también situado en la costa.

\section{ESTIMACIONES DE LA POBLACIÓN ACTUAL (ePOBa) A NIVEL MUNICIPAL: ALICANTE}

La heterogeneidad y la enorme variabilidad observada a pequeña escala entre las cifras de población del censo/ePOBa y las del Padrón en el año en que son comparables ( 1 de enero de 2002) provocaron que los intentos de modelización de dichas discrepancias no tuvieran éxito desde el punto de vista predictivo para generar $\mathrm{ePOBa}$ a nivel municipal.

Por otra parte, las soluciones ad hoc adoptadas en estos casos, tales como repartir la cifra de $e P O B a$ provincial según la estructura de población municipal del Censo de 2001 o del Padrón del año correspondiente, no son aceptables puesto que ello supone simplemente mantener una distribución de población municipal constante en el tiempo en el primer caso, o reproducir la distribución del Padrón sobre la cifra agregada de $e P O B a$, lo que en vista del gráfico 3 no parece una solución aceptable, además de generar saltos bruscos en las poblaciones de varios de los municipios más poblados. No es esta, en definitiva, la filosofía en la elaboración de la $e P O B a$ y por tanto no la seguiremos.

Sin embargo, si es posible derivar $e P O B a$ a nivel municipal una vez disponemos de poblaciones de partida a fecha 1 de enero de 2002, relativamente sencillas de obtener con la información disponible, tal y como acabamos de ver en el apartado anterior, y la información sobre flujos relevante que nos permita reconstruir los stocks de población a 1 de enero del periodo siguiente. En gran parte dicha información está disponible de forma pública con un desfase temporal inferior al año y medio, por lo que, supuesto estamos dispuestos a aceptar dicho desfase, la construcción de $e P O B a$ a nivel municipal es un simple ejercicio de reconstrucción de flujos, más un método de reparto de las discrepancias con la cifra de ePOBa provincial que publica el INE y que tomamos como dada.

A continuación efectuamos dicho ejercicio para los municipios de la provincia de Alicante. Idéntico ejercicio podría realizarse para una clasificación de la población por sexos o edades, tal y como hacen las $е P O B a$ a nivel regional, y las discrepancias ser resueltas mediante métodos de ajuste mecánico, como los ajustes bi-proporcionales RAS, pero en este trabajo nos limitaremos al total de la población a nivel municipal.

Un comentario previo. El objetivo es ofrecer $e P O B a$ a nivel municipal cuya agregación por provincias sea consistente con la cifra de $e P O B a$ a nivel provincial publicada por el INE. Ello introduce algunas restricciones en el ejercicio. Según el INE las $e P O B a$ «...son consideradas como la mejor aproximación estadística posible a la población actual residente en España, sus comunidades autónomas y sus provincias, construida con toda la información disponible en cada momento.» (INE 2009a, p.- 28, negrita en el original). El exigente calendario de publicación de la $e P O B a$ por parte del $\mathrm{INE}^{15}$ hace que, en el momento de elaboración de las mismas, no se disponga «...de información estadística completa y consolidada sobre la totalidad de los eventos demográficos ocurridos hasta el momento presente, lo que

15 Las cifras de la $е P O B a$ a fecha 1 de enero se difunden en torno al 20 de enero del mismo año. En la actualidad (26/4/2010) están disponibles las ePOBa a fecha 1 de abril de 2010, que fueron publicadas en la web del INE el 20 dicho mes. 
puede provocar eventuales desviaciones de los resultados de ePOBa con los flujos demográficos, y consecuentemente con los stocks de población, posteriormente observados.» (INE 2009a, p.- 28). Ello obliga al INE a realizar supuestos sobre todos y cada uno de los eventos demográficos que determinan la población futura a partir del stock actual, para ser capaces así de aplicar el método de componentes, aún cuando información sobre algunos de estos flujos estará disponible, con práctica certidumbre, algunos meses después. A partir de supuestos de carácter nacional, el INE efectúa ajustes en los mismos a nivel provincial para tomar en consideración comportamientos territoriales diferenciados, en una aproximación top-down a la elaboración de las ePOBa regionales. Los detalles metodológicos están descritos de forma exhaustiva en INE (2009a).

Sin duda alguna, las ePOBa serían menos actuales, pero más ajustadas a la realidad, si se decidiera esperar a que estuvieran disponibles los datos definitivos del MNP en relación a nacimientos y defunciones, así como las migraciones interiores de la Estadística de Variaciones Residenciales (EVR), y se dejaran los supuestos estimativos a la migración exterior, que en los últimos años ha constituido el componente determinante de la evolución de la población, al mismo tiempo que el más volátil y el de más difícil predicción.

Para la elaboración de la $e P O B a$ a nivel municipal adoptamos igualmente el método de componentes. Tomando como ciertas las cifras derivadas del MNP en cuanto a nacimientos y defunciones, y las de la EVR en cuanto a migraciones interiores, entendiendo por tales todas las inter-municipales, tanto dentro de la misma provincia, como las que tienen origen y destino otras provincias de España, la discrepancia entre la población resultante de la consideración de estos flujos y la cifra de $e P O B a$ publicada por el INE se debe, necesariamente, a dos causas, $(i)$ las migraciones exteriores, y/o (ii) las diferencias entre los supuestos del INE acerca de los diferentes eventos demográficos y las cifras finalmente observadas.

En concreto, dado un ámbito territorial determinado, sólo existen cuatro formas de entrar o salir de la población que vive en dicho territorio. De esta forma podemos estar seguros de que el cambio en el stock de población que habita en un territorio de referencia durante un periodo de tiempo, digamos un año, puede ser atribuido a la magnitud de estos cuatro componentes. Si el territorio de referencia es un municipio, $i$, entonces,

$$
P_{i}^{t+1}=P_{i}^{t}+\left(N_{i}^{t}-D_{i}^{t}\right)+\left(I_{i}^{t}-O_{i}^{t}\right)
$$

donde $P_{i}^{t}$ y $P_{i}^{t+1}$ son los stocks de población al comienzo de los años $t$ y $t+1$, respectivamente, $N_{i}^{t}, D_{i}^{t}, I_{i}^{t}$ y $O_{i}^{t}$ son, respectivamente, los nacimientos, las defunciones, el flujo de inmigraciones (entradas) y el flujo de emigraciones (salidas) durante el año $t$ con referencia el municipio $i$.

Información a nivel municipal para los componentes $N_{i}^{t}$ y $D_{i}^{t}$ está disponible a partir de los datos del MNP. El componente $I_{i}^{t}$, inmigraciones con destino el municipio $i$, puede desglosarse en tres componentes según su procedencia: $(i)$ otro municipio de la misma provincia, $I M_{i}^{t}$, (ii) un municipio de distinta provincia, $I P_{i}^{t}$, y (iii) el extranjero, $I E_{i}^{t}$. De idéntica forma, el componente $O_{i}^{t}$, emigraciones de procedencia el municipio $i$, puede desglosarse en tres componentes según su destino: $(i)$ otro municipio de la misma provincia, $O M_{i}^{t}$, (ii) un municipio de distinta provincia, $O P_{i}^{t}$, y (iii) el extranjero, $O E_{i}^{t}$. De esta forma el saldo migratorio del municipio $i$ puede descomponerse en la suma de tres saldos migratorios, 


$$
I_{i}^{t}-O_{i}^{t}=\left(I M_{i}^{t}-O M_{i}^{t}\right)+\left(I P_{i}^{t}-O P_{i}^{t}\right)+\left(I E_{i}^{t}-O E_{i}^{t}\right)
$$

Información a nivel municipal sobre el origen y el destino de las migraciones no está disponible por parte de la información elaborada INE y sólo es posible la identificación, a partir de los microdatos de la EVR, para aquellos municipios mayores de 10.000 habitantes. Sin embargo para este ejercicio una identificación completa de los componentes que aparecen en la parte derecha de la ecuación (2) fue suministrada por el Instituto Valenciano de Estadística (IVE).

Por tanto, tomando como punto de partida la $e P O B a$ a nivel municipal del cuadro $5, P_{i}^{2002}, \mathrm{y}$ los flujos del MNP y de la EVR podemos generar $e P O B a$ para los años sucesivos para todos los municipios de la provincia de Alicante.

Como ya hemos mencionado, si las poblaciones así generadas a nivel provincial, agregando para todos los municipios, no coinciden con las cifras de la $e P O B a$ del INE ello puede deberse a dos causas: $(i)$ a los supuestos introducidos en la elaboración de la ePOBa por parte de INE para ofrecer poblaciones con el menor desfase temporal posible, y/o (ii) que los componentes no estén correctamente medidos.

En este sentido es conocido que «... la estimación de los flujos de emigración exterior constituye hasta la actualidad el punto más frágil de la estadística demográfica española debido, fundamentalmente, a que los Padrones Municipales presentan un claro subregistro en lo que respecta a la emigración al extranjero de individuos de nacionalidad extranjera...» INE (2009a, p.-178). ${ }^{16}$ Así pues, podemos suponer que todos los componentes son razonablemente medidos con excepción del término $\left(I E_{i}^{t}-O E_{i}^{t}\right)$ en (2).

En cualquier caso, la obtención de $P_{i}^{t+1}$ para los años de 2003 a 2008 a partir de la ecuación (1) proporcionó excelentes resultados, en el sentido de que las discrepancias a nivel provincial con la cifra de $e P O B a$ publicada por el INE fue muy reducida. Tal y como muestra el cuadro 6, la discrepancia absoluta nunca superó los 10.000 habitantes, lo que en términos relativos supone siempre discrepancias inferiores al 0.6\%. En algunos años, por ejemplo 2005, esta discrepancia fue extremadamente reducida. La conclusión lógica es que este ajuste por flujos anuales, a partir de las poblaciones municipales del Censo de 2001, una vez hemos repartido las discrepancias, puede tomarse razonablemente como ePOBa nivel municipal, al menos para la provincia de Alicante.

Cuadro 6

ePOBa ALICANTE: AJUSTADA POR FLUJOS DEL PERIODO VERSUS CIFRA INE

\begin{tabular}{|c|c|c|c|c|c|c|c|}
\hline & \multicolumn{2}{|c|}{ ePOBa } & \multicolumn{2}{|c|}{ Discrepancia } & \multirow{3}{*}{$\begin{array}{l}\text { Padrón } \\
1^{\circ} \text { Enero }\end{array}$} & \multirow{2}{*}{\multicolumn{2}{|c|}{$\begin{array}{c}\text { Diferencia Padrón - } \\
\text { ePOBa (INE) }\end{array}$}} \\
\hline & \multirow{2}{*}{$\begin{array}{c}\text { Ajustada por (1) } \\
1^{\circ} \text { Enero }\end{array}$} & \multirow[t]{2}{*}{ INE } & \multirow{2}{*}{ Absoluta } & \multirow[t]{2}{*}{ Relativa } & & & \\
\hline & & & & & & Absoluta & Relativa \\
\hline 2003 & $1,517,962$ & $1,523,274$ & 5,312 & $0.35 \%$ & $1,632,349$ & 109,075 & $7.16 \%$ \\
\hline 2004 & $1,575,232$ & $1,583,373$ & 8,141 & $0.52 \%$ & $1,657,040$ & 73,667 & $4.65 \%$ \\
\hline 2005 & $1,647,282$ & $1,647,835$ & 553 & $0.03 \%$ & $1,732,389$ & 84,554 & $5.13 \%$ \\
\hline 2006 & $1,707,394$ & $1,716,679$ & 9,285 & $0.54 \%$ & $1,783,555$ & 66,876 & $3.90 \%$ \\
\hline 2007 & $1,777,769$ & $1,776,502$ & $-1,267$ & $-0.07 \%$ & $1,825,264$ & 48,762 & $2.74 \%$ \\
\hline 2008 & $1,835,318$ & $1,838,972$ & 3,654 & $0.20 \%$ & $1,891,477$ & 52,505 & $2.86 \%$ \\
\hline
\end{tabular}

Fuente: Movimiento Natural de la Población, Defunciones y Nacimientos. Estadística de Variaciones Residenciales. Padrón Municipal. INE (ueb) e IVE.

16 Puede verse a este respecto Fernández Cordón (2006) o Fernández Cordón y Leguina (1999). 
El cuadro 6 permite observar también como, en la mayoría de los casos, la $e P O B a$ ajustada según la ecuación (1) subestima la cifra de $e P O B a$ ofrecida por el INE. Sin embargo, si el subregistro padronal en lo que hace referencia a la población extranjera es correcto, deberíamos esperar justo lo contrario, a menos que no se haya compensado adecuadamente este subregistro en la población provincial de $e P O B a$ ofrecida por el INE.

Prácticamente cualquier criterio demográfico es razonable para el reparto de las discrepancias mostradas en el cuadro 6, dado que su reducida magnitud no supone alteraciones sustanciales en la estructura demográfica municipal respecto a la que se deriva de la aplicación de la ecuación (1). En lugar de distribuir dichas discrepancias según la población relativa municipal, que es lo más habitual en este tipo de ajustes, se ha optado por efectuar dicha distribución según la estructura de población extranjera, ya que esto combina, tanto la estructura demográfica, como la importancia relativa de la población extranjera dentro de cada municipio en relación al promedio de población extranjera provincial, y es precisamente el seguimiento de la población extranjera, dada su movilidad, la que mayores problemas causa en términos de cuantificar los efectivos demográficos.

En efecto, sea $E_{i}$ el número de extranjeros del municipio $i$ y $E=\sum_{i=1}^{n} E_{i}$ el total provincial, entonces podemos escribir,

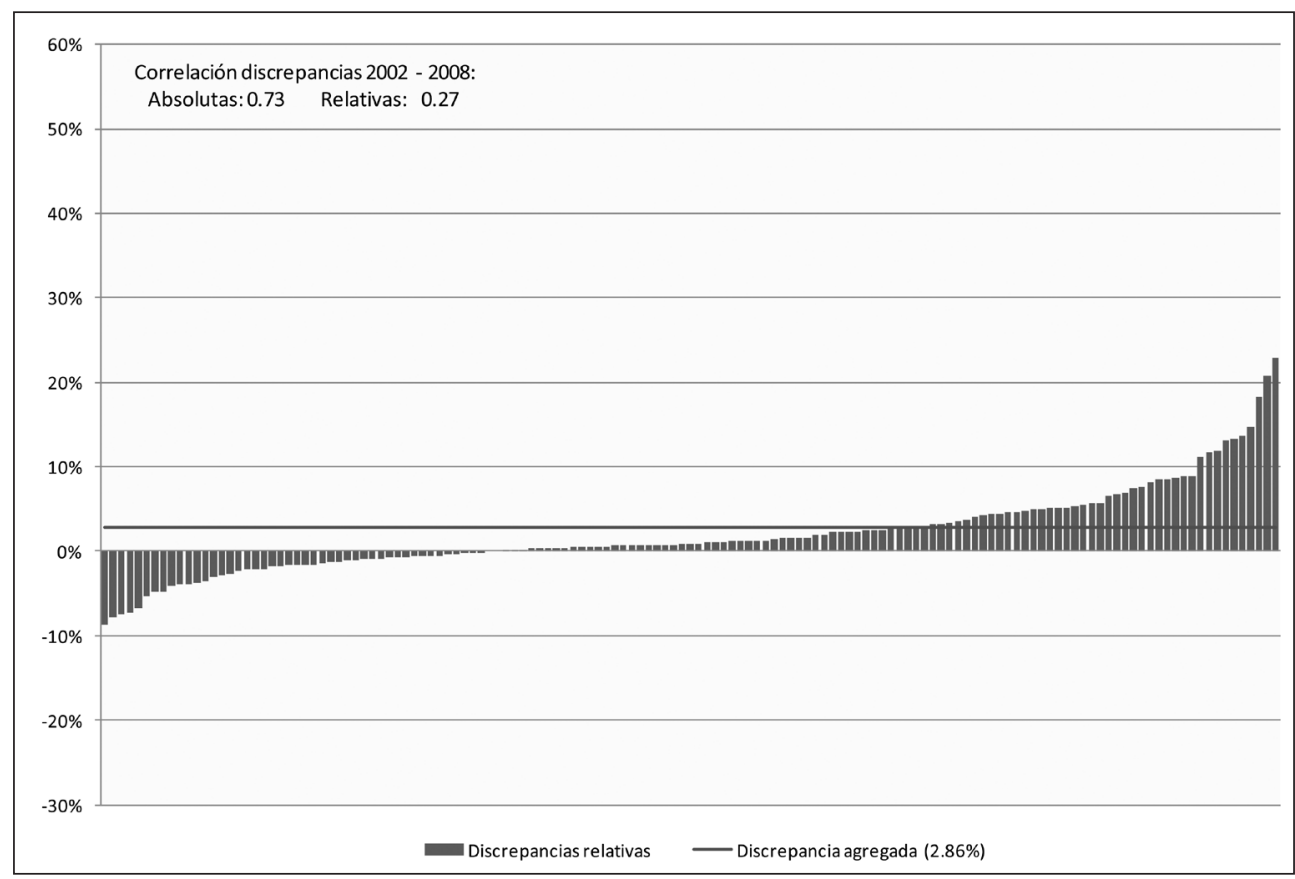

Fuente: Elaboración propia a partir de la ePOBa, el Padrón municipal 2008, el Movimiento Natural de la Población y la Estadística de Variaciones Residenciales, INE e IVE. 


$$
\frac{E_{i}}{E}=\frac{E_{i}}{P_{i}} \cdot \frac{P_{i}}{P} \cdot \frac{P}{E}=\frac{P_{i}}{P} \cdot \frac{E_{i} / P_{i}}{E / P}
$$

Así pues, la estructura de la población extranjera puede pensarse que recoge, tanto la importancia demográfica relativa del municipio dentro de la provincia, como la importancia relativa de la población extranjera dentro del propio municipio en relación al porcentaje de población extranjera de la provincia.

Las poblaciones municipales resultantes para el periodo 2002 a 2008 están disponibles si se solicitan al autor y pueden considerarse como $e P O B a$ municipales, en el sentido de que están ancladas en los niveles del Censo de 2001 y son, a su vez, consistentes con las $e P O B a$ provinciales publicadas por el INE. El gráfico 4 muestra las discrepancias relativas entre estas poblaciones y las ofrecidas por el Padrón en 2008, ordenadas de menor a mayor, y con la misma escala que en el gráfico 3, para facilitar la comparación.

$\mathrm{Al}$ contrario de lo que se observa a nivel nacional (cuadro 1), en la provincia de Alicante se ha producido un acercamiento entre las cifras de población de la ePOBa y del Padrón, tal y como se observa en el cuadro 6. A nivel municipal esta convergencia ha afectado a todos los municipios de forma homogénea, de forma que el perfil de discrepancias que ya observamos para el año 2002 se sigue manteniendo, aunque presenta una menor variabilidad.

\section{CONCLUSIONES}

Este trabajo ha examinado las discrepancias entre las cifras de población a nivel municipal del Censo de 2001 y del Padrón Municipal de habitantes. Contrariamente a lo que sucede a nivel agregado, a escala municipal el Padrón no ofrece siempre una cifra de población más elevada, lo que ya comenzaba a ser visible a nivel provincial. A pequeña escala la disparidad de cifras entre ambas fuentes de información se incrementa de forma notable. Hemos observado como estas discrepancias se correlacionan con características demográficas y físicas de los municipios, y presentan una elevada correlación espacial, ya que dichas discrepancias tienden a agruparse según patrones bien definidos.

A partir de esta observación, hemos planteado la cuestión de elaborar poblaciones municipales consistentes con las $e P O B a$ a nivel provincial publicadas por el INE. Dado un stock inicial de población municipal a 1 de enero de 2002, proveniente de ajustar las cifras del Censo de noviembre de 2001 con el crecimiento vegetativo observado en el MNP, la ecuación dinámica (1) proporciona resultados excelentes a partir de los flujos de nacimientos y defunciones del MNP y de migraciones de la EVR, en el sentido de que la discrepancia entre la población estimada de esta forma para Alicante y la cifra de $e P O B a$ ofrecida por el INE es muy pequeña para todos los años considerados. Una vez repartida esta discrepancia, de acuerdo con la estructura municipal de población extranjera, disponemos de cifras de $e P O B a$ municipales en el sentido de que parten de los niveles del Censo de 2001 y al mismo tiempo son consistentes con las $e P O B a$ provinciales publicadas por el INE. El método es sencillo, y podría generalizarse sin excesivos problemas a una clasificación de la población por sexos y edades. 
No obstante, la bondad de estas ePOBa a cualquier nivel de agregación geográfica sólo podrá ser juzgada a partir de las cifras del próximo Censo de 2011, y cuya metodología diferirá ligeramente de la de los censos anteriores. ${ }^{17}$

\section{REFERENCIAS BIBLIOGRÁFICAS}

ARANGO, J. (1981): «Los censos de población españoles en perspectiva histórica». En Primeras Jornadas de Estadística Española. Madrid. Instituto Nacional de Estadística. Vol.-3, 51-72.

FERNÁNDEZ CORDÓN, J. A. (2006): «Cuántos son?», El País, 23 de Agosto de 2006.

FERNÁNDEZ CORDÓN, J. A. Y LEGUINA, J. (1999): «Las incoherencias del nuevo padrón de habitantes», El País, 5 de julio de 1999.

GARCÍA COLL, A. Y SÁNCHEZ AGUILERA, D. (2001): «Las estadísticas demográficas españolas: Entre el orden y el caos», Boletín de la Asociación de Geógrafos Españoles, 31, 87-109. [http://www.ieg.csic.es/Age/boletin/31/3105.pdf].

GOERLICH, F. J. (2007): «¿Cuántos somos? Una excursión por las estadísticas demográficas del Instituto Nacional de Estadística (INE)», Boletin de la A.G.E., 45, $3^{\text {er }}$ cuatrimestre, 123-156. [http://age.ieg.csic.es/boletin/45/06-cuantos.pdf].

GOERLICH, F. J. Y CANTARINO, I. (2010): «Un índice de rugosidad del terreno a escala municipal a partir de Modelos de Elevación Digital de acceso público». Documento de Trabajo 7-2010, Fundación BBVA. Bilbao.

GOERLICH, F. J.; MAS, M.; AZAGRA, J. Y CHORÉN, P. (2006): La Localización de la Población Española sobre el Territorio. Un siglo de Cambios. Un Estudio Basado en Series Homogéneas (1900 - 2001). Bilbao. Fundación BBVA.

I.N.E. (2006): Avance del Padrón Municipal a 1 de enero de 2006. Datos provisionales. July-25 ${ }^{\text {th }}, 2006$. Nota de Prensa. [http://www.ine.es/prensa/np421.pdf].

I.N.E. (sin fecha): Estimaciones de la Población Actual (ePOBa). Metodología detallada. Metodología vigente hasta el 1 de enero de 2009. [http://www.ine.es/daco/daco43/epoba/ metodo.pdf].

I.N.E. (2009a): «La medición estadística de la población residente en España: Las Estimaciones de la Población Actual. Unidad didáctica del curso de la Escuela de Estadística de las Administraciones Públicas», Madrid, 21 y 22 de mayo de 2009.

I.N.E. (2009b): Estimaciones de la Población Actual (ePOBa). Metodología detallada. (Enero). Metodología vigente hasta el 1 de enero de 2009. [No disponible actualmente en la web].

I.N.E. (2010): Estimaciones de la Población Actual (ePOBa). Metodología. Metodología vigente a partir de 2009. (Abril). [http://www.ine.es/metodologia/t20/t2030259.pdf].

I.N.E. (2011): Proyecto de los Censos Demográficos 2011. Subdirección General de Estadísticas de la Población. (Febrero). [http://www.ine.es/censos2011/censos2011_proyecto. pdf].

MELÓN, A. (1951): «Los censos de población en España (1857-1940)», Estudios Geográficos, 43, 203-281.

17 http://www.ine.es/censos2011/censos2011_proyecto.pdf (consultado el 10/03/2011). 
PUYOL, R. (1997, ED.): Dinámica de la Población en España. Cambios Demográficos en el Último Cuarto del Siglo XX. Madrid. Editorial Síntesis.

REHER, D. S. (1997): «Fuentes para el estudio de la población», en Rafael Puyol (ed.). 21-46.

REHER, D. S. Y VALERO, A. (1995): «Fuentes de información demográfica en España», Cuadernos Metodológicos 13. Madrid. Centro de Investigaciones Sociológicas. 